\title{
Cell Wall Proteins of Staphylococcus aureus Are Involved in Cross Reactivity with Murine Cytokine Assays
}

\author{
Mehak Gull $^{1}$, Abida Bano ${ }^{1}$, Numan Javed ${ }^{1}$ \\ ${ }^{1}$ Department of Microbiology \& Molecular Genetics (MMG), University of the Punjab, Quaid e Azam (new) Campus, Lahore,
} Pakistan

\author{
DOI: $10.36348 /$ sjpm.2021.v06i03.001 \\ | Received: 26.01.2021 | Accepted: 10.02.2021 | Published: 06.03.2021 \\ *Corresponding author: Numan Javed
}

\section{Abstract}

Staphylococcus aureus is one of the important pathogen often termed as superbug. After S. aureus interacting with host innate immune system, it induces the release of pro- inflammatory cytokines like TNF- $\alpha$ and IL- $1 \beta$ from Immune cells. In the current study, various strains of $S$. aureus and their cell wall lysates indicated cross reactivity with murine cytokines ELISA antibodies. In cell wall lysates of $S$. aureus, various proteins are involved in this cross reactivity phenomenon. This cross reactivity was exhibited in the form of unusual exaggerated signals while performing ELISA for quantification of murine cytokines. The proteins of cell wall lysates among various strains of $S$. aureus were determined using SDS-PAGE. To all of our data, it is revealed that unknown epitopes of $S$. aureus are involved in producing exaggerated signals of cytokines during their quantification by ELISA. This was generated not only during interaction of various strains of $S$. aureus with murine bone marrow derived macrophages (BMDM) but also in control settings where cell free system was used in ruling out this cross reactivity phenomenon.

Keywords: S. aureus, MRSA, IL-1 $\beta$, TNF- $\alpha$, Macrophages, Cell wall lysate.

Copyright (C) 2021 The Author(s): This is an open-access article distributed under the terms of the Creative Commons Attribution 4.0 International License (CC BY-NC 4.0) which permits unrestricted use, distribution, and reproduction in any medium for non-commercial use provided the original author and source are credited.

\section{INTRODUCTION}

Staphylococcus aureus is an opportunistic pathogen that colonizes among $20 \%$ of human population. Its surface contains virulence factors in the form of cell wall anchored proteins [1]. In Epithelium of human anterior nares, Staphylococcus aureus is present. Healthy individuals are carriers of Staphylococcus aureus [2]. Bacteremia caused by $S$. aureus has $82 \%$ mortality. It is also involved in deep sealed infections and necrotizing pneumonia [3]. To lyse host cells at low concentration, $S$. aureus consists of pore forming toxins [4]. It also found to cause postsurgical infections. Elective surgery has high risk to possess this pathogen [5]. In food borne diseases, US reported that $S$. aureus also present and contaminate the food products with symptoms of Diarrhea, abdominal cramp and vomiting. It is one of the important food borne pathogen [6].

Methicillin resistant S. aureus (MRSA) is an important antibiotic resistant strain of Staphylococcus aureus. Prolonged hospitalizations, antibiotic use and intensive care admission are commonly associated with
MRSA infections. MRSA is responsible to cause meningitis, empyema and lung abscess [7]. Among neonatal intensive care units (NICU), MRSA is more prevalent. It colonizes in infants and acts as reservoir for other infants [8]. MRSA is a multi-drug resistant organism (MDRO). It is one of the cause of public health problem. According to reports, transport employees are at high risk to carry MRSA [9]. In nursing homes, MRSA spreads ideally at these places, people are living close to each other [10]. In MRSA, a mobile genetic element Staphylococcal chromosomal cassette (SCC) mec that carry both mecA and mecC gene. It encodes novel penicillin binding protein (PBP2a) [11]. Virulent determinants like Panton valentine leucocidin, phenol soluble modulins (PSMs) are also present in MRSA [12]. In healthy individuals, MRSA infections were reported without symptoms [13]. MRSA and MSSA are distinguished on the basis of antibiotic susceptibility. The minimum resistance in $S$. aureus has occurred due to mutation in a chromosome encoded penicillin binding protein (PBP2a) [7]. During war injuries, high consumption of antibiotics, lack of hygiene environment and overcrowding MRSA infections occurs. Healthcare 
professionals are important sources of MRSA infections [14]. Recent reports from clinical isolates of wounds and blood suggested that there is a need to develop good quality laboratories that isolate bacteria from clinical samples [15].

There are two types of immune system, adaptive and innate immune system. Innate immune system is the first line of defense that coordinates with adaptive immune response. Innate and adaptive immune response consists of various components. NK cells, macrophages, monocytes and dendritic cells are important components of innate immune response. The pathogen associated molecular patterns present on pathogen interacts with patter recognizing receptors present on immune cells. Both receptor and ligand binds and activates a series of immune cells of host. This recognition further activates pro-inflammatory cytokines, TLRs and other factors [16]. Inflammosome is a multi-protein entity that is activated by Toll like receptors TLRs and ALRs. It activates pro-caspase into caspase- 1 that play important role during inflammation and induce pro-inflammatory cytokines TNF-alpha and IL-1beta [17]. The immune response helps in determining microbial location and pathogenicity [18]. Innate immune response is such type of immune response that provides homeostasis during physical and chemical barriers. During infection that caused by pathogen, it provides protection to external structures [19]. When foreign pathogen enters, innate immune cells first encounter it. This entry of pathogen activates innate immune response and immune system within seconds respond to the infectious microorganism but adaptive immune system required time for activation [20]. Staphylococcus aureus recognized by pattern recognizing receptors (PRRs). For recognition of staphylococcal cell wall component TLR2 is specific that identify major components lipoprotein and peptidoglycan [11]. S. aureus evades host immune responses, by using its virulence factors that facilitate disease production. High level of cytolysins are also produced by this pathogen [21]. NLRP3 exists in NLR family of pattern recognizing receptors. This member NLRP3 combines with adapter protein (ASC) and causes the activation of caspase- 1 by inflammasome assembly. On the entry of pathogen immune response cooperates with each other [22].

The present study describes that $S$. aureus cell wall proteins are involved in cross reactivity with murine cytokines. $S$. aureus has ability to cross react with murine cytokines ELISA antibodies. To determine this, cell wall lysates were prepared to infect the mouse BMDM in addition to whole MRSA and MSSA. The level of pro-inflammatory cytokines like TNF- $\alpha$ and IL$1 \beta$ in cell culture supernatants were also determined by ELISA. In parallel with infected murine BMDMs, a cell free system, without BMDMs containing only whole bacteria and bacterial cell wall lysates, also exhibited the exaggerated level of cytokines. Moreover, this study revealed the involvement of $S$. aureus cell wall protein in cross reactivity with murine cytokines assays. It also provided a new direction for better understanding of immune response against $S$. aureus. It elaborated the role of $S$. aureus cell wall protein as a cross reactive molecule.

\section{MATERIALS AND METHODS Bacterial Strains Characterization}

Nasal swab samples were collected from eight healthy individuals of normal population. Among bacterial isolates of nasal swabs, four were as MRSA and rest four were MSSA. Those strains were grown in tryptic soybean casein digest medium (TSB) at $37^{\circ} \mathrm{C}$. Isolated $S$. aureus strains were characterized through biochemical tests. MRSA strains were designated as MR12, MR13, MR18 and MR70 while MSSA strains were referred as MS1, MS2, MS3 and MS4.

\section{Antibacterial susceptibility testing}

According to CLSI, by using Kirby-Bauer disc diffusion method, antimicrobial susceptibility of $S$. aureus strains were also determined [23]. The two antibiotics like oxacillin and cefoxitin were used to check the sensitivity of those strains on Mueller-Hinton agar.

\section{Preparation of cell wall lysate}

Cell wall lysates were prepared according to Wyke's protocol [24]. Briefly, the strains of $S$. aureus were cultured in appropriate medium. After that strains were sub cultured to obtain specified cells density using McFarland standard. At $600 \mathrm{~nm}$ the optical density of bacteria was adjusted to 0.257 . The culture was centrifuged in refrigerated centrifuge at $10,000 \mathrm{rpm}$ for $20 \mathrm{~min}$. The strains were washed with PBS buffer and then sonicated in order to prepare cell wall lysate. After sonication, cell wall lysates were suspended in Non idet NP40 and potassium phosphate buffer. The complete lysis of bacterial isolates was evaluated by culturing of cell wall lysate preparation on MSA agar. The absence of growth on MSA agar indicated the complete cell wall preparation.

\section{Preparation and infection of murine BMDM}

BMDMs were prepared according to protocol described in [25]. From healthy wild type mouse leg bones (Tibias and femurs), bone marrow cells were flushed out and cultured in specified medium IMDM supplemented with $10 \%$ FBS and GM-CSF (20 ng/ml). BMDM were incubated for 5 days in a humidified incubator with $5 \% \mathrm{CO}_{2}$ at $37^{\circ} \mathrm{C}$. In 96 well plates, mature and differentiated BMDM were plated for overnight. Those mature BMDM were then infected with whole bacteria and cell wall lysates of both (MRSA 12,13,18,70 and MSSA 1,2,3,4 respectively) at multiplicity of infection (MOI) 1 for overnight. After overnight incubation, supernatants from infected 
BMDMs were collected and used for further analysis in the study.

\section{Cell free system for whole bacteria and their cell wall preparation}

In this system, only RPMI medium with $10 \%$ FBS but without penicillin and streptomycin was used lacking BMDM. That system was inoculated with whole bacterial cells and their respective cell wall lysates for overnight. System was run in parallel with the BMDM medium in order to rule out phenomenon of cross reactivity.

\section{ELISA}

The quantification of cytokines like mouse TNF- $\alpha$ and IL- $1 \beta$ were determined through ELISA kit instructions (Ebioscience, USA). Those ELISA kits were used to quantify murine cytokines induced by bacterial infection in BMDM. Similarly, in cell free system (containing only bacterial cells), the level of cytokines were also identified.

\section{SDS-PAGE}

The cell walls lysates of MRSA and MSSA were analyzed by sodium dodecyl acrylamide gel electrophoresis. The protocol to perform SDS-PAGE mentioned in (SDS-PAGE BIORAD). Sampleso f $S$. aureus cell wall lysates (whole strains pellet) were

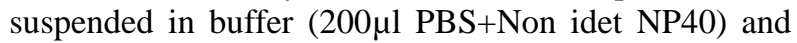
$5 \mu 1$ of loading dye was added in each strain. Samples were subjected to heat shock at $95^{\circ} \mathrm{C}$ for 5 minutes. The resolving gel $(15 \%)$ and $2 \mathrm{ml}$ stacking gel were prepared and allowed to polymerize for 30 to 45 minutes. Gel was poured in casting tray and SDS-PAGE BIORAD apparatus filled with gel running buffer. Samples of cell wall lysates were loaded in each well along with commercially prepared (pre stained protein). Electrophoresis chamber was connected with power supply for $1 \mathrm{~h}$. After $1 \mathrm{~h}$, electric supply was powered off and gel was stained with staining agent as mentioned in protocol and then gel was de-stained with destainning agent for overnight. After destainning of gel, protein bands on gel was observed.

\section{RESULTS}

\section{S. aureus strains induced mouseIL-1 $\beta$ after BMDM} infection

After the infection of $S$. aureus strains (MRSA 12, 13, 18 and 70 MSSA 1, 2, 3, 4) and their cell wall lysates with BMDM, ELISA determine the level of cytokines IL-1 $\beta$. It was observed that in BMDM after infection, the whole bacteria and their cell wall lysates induced cytokines in a variable manner and each strain and cell wall lysate respond in a different way.

\section{Determination of mouse TNF- $\alpha$ induced by $S$. aureus in BMDM}

After infecting BMDM, strains of $S$. aureus and cell wall lysates also induced TNF- $\alpha$ (murine cytokines). It was observed that secreted level of TNF- $\alpha$ was low. Each MRSA and MSSA strain showed the level of cytokines in a specific range.

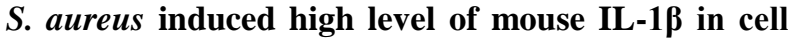 free system}

Cell free system consists of only whole bacteria of $S$. aureus strains and their cell wall lysates. That was used as a control to determine without BMDM, for the quantification of secreted cytokines.

\section{ELISA determine high level of mouse TNF-alpha in cell free system}

In the case of control, cell free system which was without murine BMDM, mouse TNF- $\alpha$ was also determined by ELISA upon inoculation with whole $S$. aureus strains and their respective cell wall preparations. Strains of $S$. aureus also induced proinflammatory cytokines TNF- $\alpha$. In BMDM, cytokines level was different as compared to cell free system.

\section{Quantification of S. aureus cell wall protein by SDS- PAGE}

$S$. aureus strains that induced proinflammatory cytokines and higher level in cell free system. The proteins that were present in cell wall lysates induced these pro-inflammatory cytokines TNF$\alpha$ and IL-1 $\beta$. For this, the cell wall lysates were analyzed by SDS-PAGE for the determination of protein concentration in cell wall lysates, Moreover, the bradford assay was also used for quantification of protein in the cell wall lysates samples (data not shown). Both MRSA and MSSA, cell lysates whole bacterial pellet with loading dye as a sample was prepared. Pre-stained ladder determined the molecular weight of protein.

\section{DISCUSSION}

This study was conducted in order to identify the involvement of Staphylococcus aureus cell wall proteins that play important role in cross reactivity of murine cytokines assays. MRSA is one of the important medical pathogen responsible for many type of infectious diseases but with the increase in resistance of antimicrobial activities, this superbug also adapted evasion mechanisms to evade the host immune response. These immune cells also develop various kinds of host immune response. When $S$. aureus invades the host, the host immune cells in response to pathogen, secrete pro-inflammatory cytokines like TNF- $\alpha$ and IL- $1 \beta$. This study has suggested that there are various unknown proteins present in cell wall of Staphylococcus aureus, responsible for this host pathogen interaction. Various MRSA and MSSA strains of $S$. aureus were selected from normal healthy individuals, we used these strains as a whole as well as their cell wall lysate preparation in order to rule out their potential involvement in cross reactivity with murine cytokine assays. 
Recently, it has been suggested that $S$. aureus cell wall proteins can be involved in cross reactivity of murine cytokines. It also has the ability to activate the pro-inflammatory cytokines like TNF- $\alpha$ and IL-1 $\beta$. $S$. aureus cross react with murine cytokines ELISA antibodies which could be source of virulence [26].

To determine the involvement of protein we selected the strains of $S$. aureus MSSA and MRSA. Cell wall lysates from these strains were prepared and using protocol, we analyze the whole strains and cell wall lysates. ELISA results have shown that MSSA among all strains induced higher level of IL- $1 \beta$ but their cell wall lysates showed less amount of IL- $1 \beta$ as shown in (fig 3.1). These findings suggest that there is a cross reactivity that has occurred and production of exaggerated pro-inflammatory cytokines. Interestingly, it was observed that as compared to MRSA, MSSA have more capability to induce cytokines in case of BMDM which is evident of base line level of cytokines from BMDM induction in addition exaggerated signals of cytokines. According to studies, for host inflammatory response, the release of IL-1 $\beta$ is necessary and it is one of the important proinflammatory agent [27]. In case of TNF- $\alpha$ (as shown in Fig-2), there were similar results like in IL-1 $\beta$. Among all strains, MS4 have higher level of TNF- $\alpha$. MS4 consists of factors (protein) which stimulates the production of pro-inflammatory cytokines and could be involved in cross reactivity with ELISA antibodies. It has been studied that in murine model of osteomyelitis, $S$. aureus has capability to induce higher level of proinflammatory cytokines TNF- $\alpha$ and IL-1 $\beta$ [28].

In Fig $1 \& 2$, we didn't observe the induction of inflammatory cytokines by MRSA cell wall lysates. It indicated that in these cell wall lysates there were no involved proteins that could cross react with proinflammatory cytokines. During $S$. aureus cell wall preparation, the absence of cross reactive signals is due to loss of potential cross reactive factor. In cell free system, we observed higher level of TNF- $\alpha$ and IL-1 $\beta$. In Fig-3, it was observed that without BMDM cells, bacterial strains induce fake signals of proinflammatory cytokines TNF- $\alpha$ and IL- $1 \beta$. Interestingly on comparison it was identified that MR strains also induce fake signals of IL- $1 \beta$ but MS3 and MS4 secretes higher level of IL-1 $\beta$.

MRSA and MSSA strains along with their cell wall lysates showed strong induction of TNF- $\alpha$. As there were no BMDM cells (Fig-4), each strain of $S$. aureus has higher potential for TNF- $\alpha$ secretion. These findings apparently indicated that it is cross reactivity but infact, these are fake or exaggerated level of cytokines. Recent studies determined that MRSA also have ability to induce higher level of pro-inflammatory cytokines. $S$. aureus showed similar level of TNF- $\alpha$ in earlier studies [26]. Our data indicated that MS4 was a strong inducer of both IL-1 $\beta$ and TNF- $\alpha$ during BMDM infection with MS4. Moreover, the exaggerated signals for both IL-1 $\beta$ and TNF- $\alpha$ were observed in cell free system with MS4 which is indicative that MS4 has the ability to cross react with pro-inflammatory cytokine ELISA assays.

To induce pro-inflammatory cytokines, according to observation there were some factors that were responsible. For this purpose, we analyzed bacterial cell wall lysates by SDS-PAGE because at this stage we were not sure that it was protein in crude extract cell wall lysates. As in Fig $1 \& 2$, there were no induction of cytokines by cell wall lysates, it could be possible that proteins in cell lysate were degraded. In order to confirm the presence of proteins in cell wall extract, the Bradford assay was used to measure concentrate of protein in samples using bovine serum albumin as a standard [29].

We observed bands of $S$. aureus proteins having molecular weight in range of 72 to $55 \mathrm{kDa}$. We observed bands of MR13, 18 and MR70. In (Fig-5), MS1 and MS2 showed bands but in MS3 and MS4, there were no bands. In comparison of both SDS PAGE and ELISA results, we observed that the $S$. aureus strains showed protein bands on SDS PAGE and that protein could be responsible for cross reactive signals of pro-inflammatory cytokines. Rather than MRSA, MSSA also have ability to generate high level of proinflammatory cytokines like IL- $1 \beta$ and TNF- $\alpha$. Earlier studies also identified proteins from cell wall lysates by using both techniques ELISA and SDS PAGE [30].

MOI of bacteria is the main cause of proinflammatory cytokines induction. In dose dependency, there is a contradiction that use of antimicrobial agents started to kill bacteria instead of preventing host response [31]. For infection, dose is important. Our data suggested that bacterial strains and their cell wall lysates cause the release of pro-inflammatory cytokines and protein in the cell wall lysates acts as a cross reactive molecule. However, according to reported data, $S$. aureus has involved in NLRP3 inflammosome activation [32].

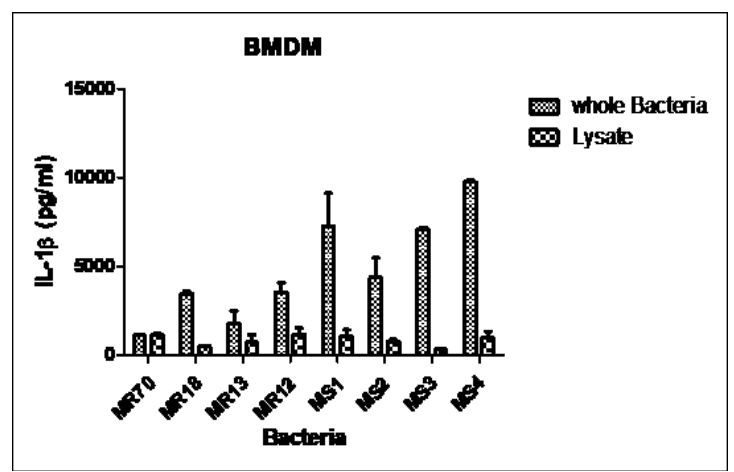

Fig-1: Determination of mouse IL-1及, after infecting murine BMDM with whole bacterial strains and lysates 


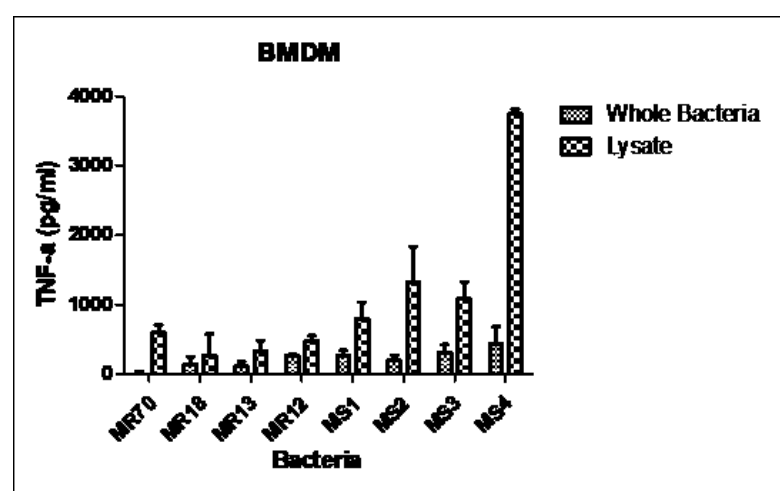

Fig-2: Determination of mouse TNF- $\alpha$, after infection of BMDM with $S$. aureus strains and their lysates

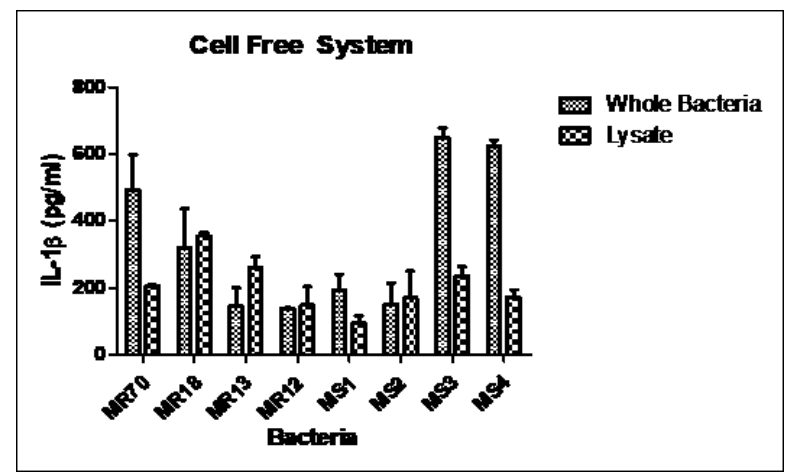

Fig-3: In cell free system without BMDM culture cells, only whole bacterial cells and their lysates were inoculated and supernatant was estimated for mouse IL-1 $\beta$ by ELISA

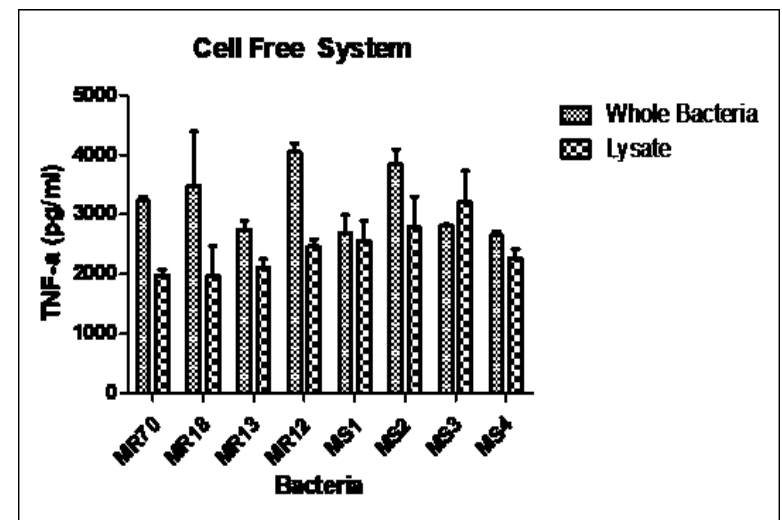

Fig-4: Determination for TNF- $\alpha$, each strain of Whole bacteria MRSA and MSSA with their lysates in ELISA

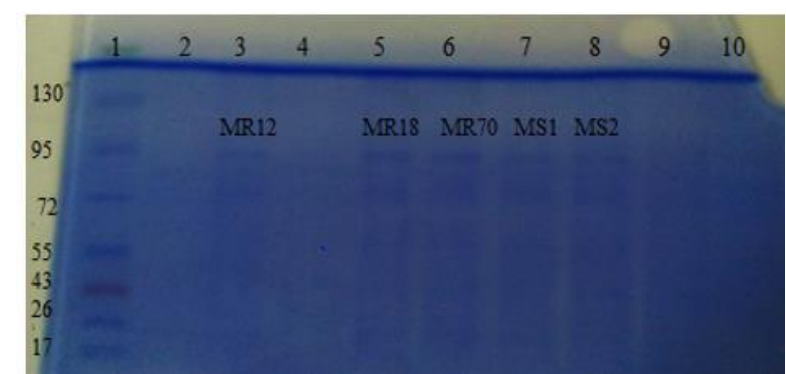

Fig-5: Lane 1. Ladder; 2. Pooled sample MR 12; 3. Pellet MR 12; 4. Pellet MR 13; 5. Pellet MR 18; 6. Pellet MR 70; 7. Pellet MS 1; 8. Pellet MS2; 9. Pellet MS3; 10. Pellet MS4

\section{CONCLUSION}

The present study was designed to test the hypothesis that $S$. aureus has ability to cross react with murine cytokines assays. As $S$. aureus have some unknown epitopes that cross react with ELISA antibodies used for murine cytokine assays. Our data indicated that upon host pathogen interaction there is cross reactive molecule present expectedly in the cell wall of pathogen to interact with the pro-inflammatory cytokines. In conclusion, our study indicates that $S$. aureus strains and their cell wall lysates consists of various proteins that could cross react with murine cytokines. Various assay techniques also verified the presence of protein and their involvement as a cross reactive molecule. In future further identification and purification of those cross reactive molecule will be helpful to explain their role and significance. Moreover, it would also be able to understand the host immune evasion strategies by $S$. aureus.

\section{ACKNOWLEDGEMENT}

We highly acknowledge Higher Education Commission (HEC) of Pakistan for providing funding for this research work.

\section{REFERENCES}

1. Foster, T. J., Geoghegan, J. A., Ganesh, V. K., \& Höök, M. (2014). Adhesion, invasion and evasion: the many functions of the surface proteins of Staphylococcus aureus. Nature reviews microbiology, 12(1), 49-62.

2. Foster, T. J. (2004). The Staphylococcus aureus "superbug". The Journal of clinical investigation, 114(12), 1693-1696.

3. Thomer, L., Schneewind, O., \& Missiakas, D. (2016). Pathogenesis of Staphylococcus aureus bloodstream infections. Annual Review of Pathology: Mechanisms of Disease, 11, 343-364.

4. Seilie, E. S., \& Wardenburg, J. B. (2017, December). Staphylococcus aureus pore-forming toxins: The interface of pathogen and host complexity. In Seminars in cell \& developmental biology (Vol. 72, pp. 101-116). Academic Press.

5. Jorgensen, S., Zasowski, E. J., Trinh, T. D., Lagnf, A. M., Bhatia, S., \& Rybak, M. J. (2018, November). 1227. Development of a Clinical Prediction Model for Mortality in MethicillinResistant Staphylococcus aureus Bacteremia. In Open Forum Infectious Diseases (Vol. 5, No. suppl_1, pp. S372-S372). US: Oxford University Press.

6. Kadariya, J., Smith, T. C., \& Thapaliya, D. (2014). Staphylococcus aureus and staphylococcal food-borne disease: an ongoing challenge in public health. BioMed research international, 2014.

7. Siddiqui, A. H., \& Koirala, J. (2019). Methicillin resistant Staphylococcus aureus (MRSA). StatPearls [Internet]. 
8. Ericson, J. E., Popoola, V. O., Smith, P. B., Benjamin, D. K., Fowler, V. G., Clark, R. H., \& Milstone, A. M. (2015). Burden of invasive Staphylococcus aureus infections in hospitalized infants. JAMA pediatrics, 169(12), 1105-1111..

9. Schablon, A., Kleinmüller, O., Nienhaus, A., \& Peters, C. (2018). MRSA prevalence among patient transport staff in Hamburg. GMS hygiene and infection control, 13 ..

10. Hughes, C., Tunney, M., \& Bradley, M. C. (2013). Infection control strategies for preventing the transmission of meticillin- resistant Staphylococcus aureus (MRSA) in nursing homes for older people. Cochrane database of systematic reviews, (11).

11. Liu, J., Chen, D., Peters, B. M., Li, L., Li, B., Xu, Z., \& Shirliff, M. E. (2016). Staphylococcal chromosomal cassettes mec (SCCmec): a mobile genetic element in methicillin-resistant Staphylococcus aureus. Microbial pathogenesis, 101, 56-67.

12. Kumar, P. (2012). Recent Update on MRSA. BAOJ Microbio.

13. Amit, L. N., Yung, C. V. L., Moy, F. S., \& John, D. V. (2018). Molecular characteristics of infection and colonization isolates of communityacquired methicillin-resistant Staphylococcus aureus (CA-MRSA). Trop. Biomed, 35, 442-452.

14. Andersen, B. M. (2019). MRSA Prevention, in Prevention and Control of Infections in Hospitals, Springer, 681-711.

15. Venkatesh, V. (2018). Staphylococcus aureus and MRSA: Do we know the true burden?. Clinical Epidemiology and Global Health, 6(3), 103-104.

16. Portou, M. J., Baker, D., Abraham, D., \& Tsui, J. (2015). The innate immune system, toll-like receptors and dermal wound healing: a review. Vascular pharmacology, 71, 31-36.

17. Kanneganti, T. D. (2015). The inflammasome: firing up innate immunity. Immunological reviews, 265(1), 1.

18. Iwasaki, A., \& Medzhitov, R. (2015). Control of adaptive immunity by the innate immune system. Nature immunology, 16(4), 343-353.

19. Riera Romo, M., Pérez Martínez, D., \& Castillo Ferrer, C. (2016). Innate immunity in vertebrates: an overview. Immunology, 148(2), 125-139.

20. Semple, J. W., \& Freedman, J. (2010). Platelets and innate immunity. Cellular and Molecular Life Sciences, 67(4), 499-511.

21. Stach, C. S., Herrera, A., \& Schlievert, P. M. (2014). Staphylococcal superantigens interact with multiple host receptors to cause serious diseases. Immunologic research, 59(1-3), 177-181.

22. Jin, C., \& Flavell, R. A. (2010). Molecular mechanism of NLRP3 inflammasome activation. Journal of clinical immunology, 30(5), 628-631.

23. Anand, K. B., Agrawal, P., Kumar, S., \& Kapila, K. (2009). Comparison of cefoxitin disc diffusion test, oxacillin screen agar, and PCR for mecA gene for detection of MRSA. Indian journal of medical microbiology, 27(1), 27.

24. Wyke, A. W. (1984). Isolation of five penicillinbinding proteins from Staphylococcus aureus. FEMS microbiology letters, 22(2), 133138.

25. Meng, G., Zhang, F., Fuss, I., Kitani, A., \& Strober, W. (2009). A mutation in the Nlrp3 gene causing inflammasome hyperactivation potentiates Th17 cell-dominant immune responses. Immunity, 30(6), 860-874.

26. Javed, N., Xue, G., Lu, A., Xing, Y., Iwakura, Y., Xiao, H., ... \& Meng, G. (2016). Cross reactivity of S. aureus to murine cytokine assays: A source of discrepancy. Cytokine, 81, 101-108.

27. Giuliani, A. L., Sarti, A. C., Falzoni, S., \& Di Virgilio, F. (2017). The P2X7 receptorinterleukin-1 liaison. Frontiers in pharmacology, 8, 123.

28. Wu, T., Weng, Z., Xu, J., Wen, G., Yu, Y., \& Chai, Y. (2018). Baicalin alleviates osteomyelitis by regulating TLR2 in the murine model. Pathogens and disease, 76(2), ftx123.

29. Ernst, O., \& Zor, T. (2010). Linearization of the Bradford protein assay. Journal of visualized experiments: JoVE, 2(38), 1918

30. Pietrocola, G., Nobile, G., Gianotti, V., Zapotoczna, M., Foster, T. J., Geoghegan, J. A., \& Speziale, P. (2016). Molecular interactions of human plasminogen with fibronectin-binding protein B (FnBPB), a fibrinogen/fibronectinbinding protein from Staphylococcus aureus. Journal of Biological Chemistry, 291(35), 18148-18162.

31. Son, S. J., Park, M. R., Oh, S., \& Kim, Y. (2015). The Herbal derived Honokiol and Magnolol Enhance Host Immune Response to Infection with Methicillin sensitive Staphylococcus aureus (MSSA) and Methicillin resistant $S$. aureus (MRSA). The FASEB Journal, 29, 853-2.

32. Jo, E. K., Kim, J. K., Shin, D. M., \& Sasakawa, C. (2016). Molecular mechanisms regulating NLRP3 inflammasome activation. Cellular \& molecular immunology, 13(2), 148-159. 